\title{
Nonvanishing of twists of $L$-functions attached to Hilbert modular forms
}

\author{
Nathan C. Ryan, Gonzalo Tornaría and John Voight
}

\begin{abstract}
We describe algorithms for computing central values of twists of $L$-functions associated to Hilbert modular forms, carry out such computations for a number of examples, and compare the results of these computations to some heuristics and predictions from random matrix theory.
\end{abstract}

\section{Introduction}

Let $f(q)=\sum_{n=1}^{\infty} a_{n} q^{n} \in S_{k}(N)$ be a classical newform of weight $k$ and level $N$, and let $\lambda_{n}=a_{n} / \sqrt{n}^{k-1}$. For $D<0$ a fundamental discriminant, let

$$
L\left(f, s, \chi_{D}\right)=\sum_{n=1}^{\infty} \chi_{D}(n) \frac{\lambda_{n}}{n^{s}}
$$

be the $L$-series of $f$ (in the analytic normalization) twisted by the quadratic character $\chi_{D}$ associated to the imaginary quadratic field $\mathbb{Q}(\sqrt{D})$. The central values $L\left(f, \frac{1}{2}, \chi_{D}\right)$ encode interesting arithmetic information about the form $f$, and a number of explicit investigations have been carried out examining the family of these values $[3,15,24,27,28]$.

An efficient way to compute the family $L\left(f, 1 / 2, \chi_{D}\right)$ of central values with varying discriminant $D$ is to use Waldspurger's theorem [43], which asserts that the values are related to the Fourier coefficients of a certain half-integer weight modular form. Concretely, for $D<0$ coprime to $N$, we have

$$
L\left(f, 1 / 2, \chi_{D}\right)=\kappa_{f} \frac{c_{|D|}(g)^{2}}{\sqrt{|D|}^{k-1}}
$$

where the (nonzero) constant $\kappa_{f}$ is independent of $D$ and $c_{|D|}(g)$ is the $|D|$ th coefficient of a modular form $g$ of weight $(k+1) / 2$ related to $f$ via the Shimura correspondence. Computing central values using (1.1) has the advantage that the description of $g$ as a linear combination of theta series permits the rapid computation of a large number of coefficients: for example, HartTornaría-Watkins [17] compute hundreds of billions of twists of the congruent number elliptic curve (using FFT methods). By comparison, experiments with the distribution of twists with similar Hodge data computed without using Waldspurger's theorem are much less extensive: see for example, Watkins $[45, \S 6.6]$ and David-Fearnley-Kisilevsky [6].

Several authors have pursued Waldspurger's theorem in the setting of Hilbert modular forms, including Shimura [35], Baruch-Mao [1], Xue [46], Sirolli [38], and Hiraga-Ikeda [18]. In this paper, we develop algorithms using these formulas to compute families of central values

Received 27 February 2014; revised 23 May 2014

2010 Mathematics Subject Classification 11F41, 11M50 (primary), 11F67 (secondary).

Contributed to the Algorithmic Number Theory Symposium XI, GyeongJu, Korea, 6-11 August 2014.

The first and second authors were supported by grant CSIC I + D 2012-628 from the Comisión Sectorial de Investigación Científica. The third author was supported by an NSF CAREER Award (DMS-1151047). 
$L\left(f, 1 / 2, \chi_{D}\right)$ for Hilbert modular forms $f$ over totally real fields $F$, defined in an analogous way. We believe that these computations are of independent interest, but we also use them to provide some partial evidence for conjectures concerning statistics for central values in families of twists motivated by some heuristics and refined by random matrix theory.

Let $F$ be a totally real field of degree $n=[F: \mathbb{Q}]$ with ring of integers $\mathbb{Z}_{F}$, and suppose that $F$ has narrow class number 1 . Let

$$
\mathcal{D}\left(\mathbb{Z}_{F}\right)=\left\{D \in \mathbb{Z}_{F} / \mathbb{Z}_{F}^{\times 2}: D \text { fundamental discriminant and } D \ll 0\right\}
$$

be the set of totally negative fundamental discriminants in $\mathbb{Z}_{F}$; the set $\mathcal{D}\left(\mathbb{Z}_{F}\right)$ is in canonical bijection with the complex multiplication $(\mathrm{CM})$ extensions $F(\sqrt{D})$ of $F$. For $X>0$, let

$$
\mathcal{D}\left(\mathbb{Z}_{F} ; X\right)=\left\{D \in \mathcal{D}\left(\mathbb{Z}_{F}\right):\left|N_{F / \mathbb{Q}}(D)\right| \leqslant X\right\} .
$$

To $D \in \mathcal{D}\left(\mathbb{Z}_{F}\right)$, let $\chi_{D}$ be the character associated to the quadratic extension $F(\sqrt{D})$.

Let $f$ be a Hilbert cusp form over $F$ of parallel weight $k \in 2 \mathbb{Z}_{>0}$ and level $\mathfrak{N} \subseteq \mathbb{Z}_{F}$ with rational integer Hecke eigenvalues, and let $w_{f}$ be the sign of the functional equation for $L(f, s)$. We will be interested in the number of vanishings

$$
\mathcal{N}_{f}\left(\mathbb{Z}_{F} ; X\right)=\#\left\{D \in \mathcal{D}\left(\mathbb{Z}_{F} ; X\right): \chi_{D}(\mathfrak{N})=(-1)^{n} w_{f} \text { and } L\left(f, 1 / 2, \chi_{D}\right)=0\right\}
$$

as a function of $X$; the condition that $\chi_{D}(\mathfrak{N})=(-1)^{n} w_{f}$ is equivalent to the condition that the sign of the functional equation for $L\left(f, s, \chi_{D}\right)$ is +1 , so that $L\left(f, s, \chi_{D}\right)$ vanishes to even order.

Conjecture 1.3. There exist $b_{f}, C_{f} \geqslant 0$ depending on $f$ such that as $X \rightarrow \infty$, we have

$$
\mathcal{N}_{f}\left(\mathbb{Z}_{F} ; X\right) \sim C_{f} X^{1-(k-1) / 4}(\log X)^{b_{f}}
$$

When $k=2$, the modular form $f$ is expected (and known in many cases) to correspond to an isogeny class of elliptic curves $E$ over $F$, so according to the conjecture of Birch-SwinnertonDyer, Conjecture 1.3 predicts the distribution of curves of even rank $\geqslant 2$ in CM quadratic twists of $E$ over $F$. Conjecture 1.3 generalizes the conjecture of Conrey-Rubinstein-KeatingSnaith [4] made in the case $F=\mathbb{Q}$, thinking of $L(f, s)$ as a degree $2 L$-function over $F$. The additional power $b_{f}$ of $\log X$ that appears is conjecturally related to arithmetic and geometric properties of $f$ : for example, if $F=\mathbb{Q}$ and $k=2$, then Delaunay-Watkins [7] conjecture a value for $b_{f}$ that depends on the two-torsion structure of elliptic curves in the isogeny class associated to $f$ (see $\S 4$ below for more discussion). The constant $C_{f} \geqslant 0$ is not presently understood; when $k \geqslant 6$, we predict that $C_{f}=0$.

Our second conjecture is a variant of the first and investigates a new phenomenon that arises in the context of Hilbert modular form, restricting to twists by discriminants $D \in \mathbb{Z}$; we still write $\chi_{D}$ for the quadratic character of $F(\sqrt{D})$ over $F$. Let

$$
\mathcal{N}_{f}(\mathbb{Z} ; X)=\#\left\{D \in \mathcal{D}(\mathbb{Z} ; X): \chi_{D}(\mathfrak{N})=(-1)^{n} w_{f} \text { and } L\left(f, 1 / 2, \chi_{D}\right)=0\right\}
$$

Conjecture 1.5. There exist $b_{f, \mathbb{Z}}, C_{f, \mathbb{Z}} \geqslant 0$ depending on $f$ such that as $X \rightarrow \infty$, we have

$$
\mathcal{N}_{f}(\mathbb{Z} ; X) \sim C_{f, \mathbb{Z}} X^{1-n(k-1) / 4}(\log X)^{b_{f, \mathbb{Z}}} .
$$

In Conjecture 1.5, we instead are thinking of $L(f, s)$ as a degree $2 n L$-function over $\mathbb{Q}$, and as a result, vanishing twists (with $D \in \mathbb{Z}$ ) are more rare. Put another way, the discriminants $D \in \mathbb{Z}$ are sparse among all discriminants $D \in \mathbb{Z}_{F}$, and consequently their contribution to the number of vanishing twists is scant. The power $b_{f, \mathbb{Z}}$ of $\log X$ depends on $f$ as in Conjecture 1.5 but with an additional factor coming from the expected number of primes in the factorization in $\mathbb{Z}_{F}$ of $(p)$ for a prime $p \in \mathbb{Z}$. 
REMARK 1.6. In the case of non-parallel weight $\left(k_{1}, \ldots, k_{n}\right)$, we expect Conjectures 1.3 and 1.5 hold replacing $k$ with the average of the $k_{i}$.

This paper is organized as follows. We begin in $\S 2$ by giving some background on Hilbert modular forms and their $L$-functions, and we state a version of Waldspurger's theorem for Hilbert modular forms. In $\S 3$, we make this theory algorithmic, and exhibit methods to compute a large number of central values of $L$-functions of quadratic twists over totally real fields. In $\S 4$, we describe the heuristics motivating Conjectures 1.3-1.5 and show how they might be refined using the connections between random matrix theory and $L$-functions in our context. In $\S 5$, we describe the experiments we carried out and present some data, tables and graphs. Our computations are done primarily in Magma [2]. We conclude in $\S 6$ with some remaining questions.

\section{Background and notation}

In this section, we summarize the background and introduce the notation we use throughout. General references for Hilbert modular forms include Freitag [13], van der Geer [41], and Goren [14]; for algorithmic aspects, see the survey of Dembélé-Voight [10].

\section{Hilbert modular forms}

Let $F$ be a totally real number field of degree $n=[F: \mathbb{Q}]$, discriminant $d_{F}$, ring of integers $\mathbb{Z}_{F}$, and different $\mathfrak{d}$. We assume throughout that $F$ has narrow class number 1 ; removing this hypothesis is possible but would make the exposition more technical. Let $v_{1}, \ldots, v_{n}$ be the real embeddings of $F$ into $\mathbb{R}$. Let $\mathfrak{N}$ be a nonzero ideal of $\mathbb{Z}_{F}$. Let

$$
F_{+}=\left\{a \in F: v_{i}(a)>0 \text { for all } i=1, \ldots, n\right\}
$$

and for a fractional ideal $\mathfrak{b}$ of $F$, let $\mathfrak{b}_{+}=\mathfrak{b} \cap F_{+}$. Let $\mathcal{H}=\{z \in \mathbb{C}: \operatorname{Im}(z)>0\}$ denote the upper half-plane.

A Hilbert modular form over $F$ of weight $k=\left(k_{i}\right)_{i} \in 2 \mathbb{Z}_{\geqslant 0}^{n}$ and level $\mathfrak{N}$ is a holomorphic function $f: \mathcal{H}^{n} \rightarrow \mathbb{C}$ such that for all $z=\left(z_{1}, \ldots, z_{n}\right) \in \mathcal{H}^{n}$ and all

$$
\gamma \in \Gamma_{0}(\mathfrak{N})=\left\{\gamma=\left(\begin{array}{ll}
a & b \\
c & d
\end{array}\right) \in \mathrm{GL}_{2}\left(\mathbb{Z}_{F}\right): c \in \mathfrak{N} \text { and } \operatorname{det}(\gamma) \in \mathbb{Z}_{F,+}\right\}
$$

we have

$$
f(\gamma z)=f\left(\frac{a_{1} z_{1}+b_{1}}{c_{1} z_{1}+d_{1}}, \ldots, \frac{a_{n} z_{n}+b_{n}}{c_{n} z_{n}+b_{n}}\right)=\left(\prod_{i=1}^{n} \frac{\left(c_{i} z_{i}+d_{i}\right)^{k_{i}}}{\operatorname{det} \gamma_{i}^{k_{i} / 2}}\right) f(z),
$$

where $\gamma_{i}=v_{i}(\gamma)=\left(\begin{array}{cc}a_{i} & b_{i} \\ c_{i} & d_{i}\end{array}\right)$, and such that further $f$ satisfies a condition of bounded growth (necessary only in the case $F=\mathbb{Q}$, by Koecher's principle). If $k_{i}=k$ for all $i=1, \ldots, n$, we say $f$ has parallel weight $k \in 2 \mathbb{Z}_{\geqslant 0}$. A Hilbert cusp form is a Hilbert modular form that vanishes at the cusps, elements of $\mathbb{P}^{1}(F) \hookrightarrow \mathbb{P}^{1}(\mathbb{R})^{n}$. We denote by $S_{k}(\mathfrak{N}) \subseteq M_{k}(\mathfrak{N})$ the space of Hilbert cusp forms inside the finite-dimensional space of Hilbert modular forms of weight $k$ and level $\mathfrak{N}$ over $F$.

A Hilbert modular form $f \in M_{k}(\mathfrak{N})$ admits a Fourier expansion

$$
f(z)=a_{0}+\sum_{\mu \in\left(\mathfrak{d}^{-1}\right)_{+}} a_{\mu} q^{\operatorname{Tr}(\mu z)}
$$

where $q^{\operatorname{Tr}(\mu z)}=\exp \left(2 \pi i \sum_{i=1}^{n} \mu_{i} z_{i}\right)$, analogous to the $q$-expansion of a classical modular form. If $f(z) \in S_{k}(\mathfrak{N})$, then $a_{0}=0$. 
The space $S_{k}(\mathfrak{N})$ is equipped with the Petersson inner product given by

$$
\langle f, g\rangle=\frac{1}{\operatorname{vol}\left(X_{0}(\mathfrak{N})\right)} \int_{X_{0}(\mathfrak{N})} f(z) \overline{g(z)} y^{k-2} d x d y
$$

where $X_{0}(\mathfrak{N})=\Gamma_{0}(\mathfrak{N}) \backslash \mathcal{H}^{n}, z_{i}=x_{i}+\sqrt{-1} y_{i}$ and $d x=d x_{1} \ldots d x_{n}, d y=d y_{1} \ldots d y_{n}$. Further, the spaces $M_{k}(\mathfrak{N})$ and $S_{k}(\mathfrak{N})$ are equipped with an action of pairwise commuting Hecke operators $T_{\mathfrak{n}}$, indexed by the nonzero ideals $\mathfrak{n}$ of $\mathbb{Z}_{F}$ with $\mathfrak{n}$ coprime to $\mathfrak{N}$, that are self-adjoint with respect to the Petersson inner product. These spaces are also equipped with Atkin-Lehner operators $W_{\mathfrak{p}^{e}}$ for prime powers $\mathfrak{p}^{e} \| \mathfrak{N}$. If $f \in S_{k}(\mathfrak{N})$ is a (simultaneous) eigenform, we say $f$ is normalized if $a_{(1)}=1$; then $T_{\mathfrak{n}} f=a_{\mathfrak{n}} f$ and each eigenvalue $a_{\mathfrak{n}}$ is an algebraic integer that lies in a totally real number field $K_{f}=\mathbb{Q}\left(\left\{a_{\mathfrak{n}}\right\}\right)$.

There are natural injections $S_{k}(\mathfrak{M}) \hookrightarrow S_{k}(\mathfrak{N})$ when $\mathfrak{M} \mid \mathfrak{N}$, and we define a newform to be a normalized cusp eigenform which is orthogonal to the image of any such injection with $\mathfrak{M}$ a proper divisor of $\mathfrak{N}$. For each unitary divisor $\mathfrak{q} \| \mathfrak{N}$ (so $\mathfrak{q} \mid \mathfrak{N}$ and $\mathfrak{q}$ is coprime to $\mathfrak{N} / \mathfrak{q}$ ), there is an Atkin-Lehner operator $W_{\mathfrak{q}}$ that is an involution on the new subspace of $S_{k}(\mathfrak{N})$, so that $W_{\mathfrak{q}} f=w_{\mathfrak{q}, f} f= \pm f$ for a Hecke newform $f$.

\section{Theta series}

The theory of half-integral weight modular forms was developed by Shimura in a series of papers $[33,34,36]$. Suppose that $\mathfrak{N}$ is odd (that is, $\mathrm{N}(\mathfrak{N})$ is odd). A Hilbert modular form over $F$ of half-integer weight $k \in\left(\frac{1}{2}, \ldots, \frac{1}{2}\right)+\mathbb{Z}_{\geqslant 0}^{n}$, level $4 \mathfrak{N}$, and quadratic character $\psi$ of conductor dividing $4 \mathfrak{N}$ is a holomorphic function $f: \mathcal{H}^{n} \rightarrow \mathbb{C}$ such that for all $z \in \mathcal{H}^{n}$ and all $\gamma=\left(\begin{array}{ll}a & b \\ c & d\end{array}\right) \in \Gamma_{0}(4 \mathfrak{N})$, we have

$$
f(\gamma z)=\psi(d) h(\gamma, z)\left(\prod_{i=1}^{n} \frac{\left(c_{i} z_{i}+d_{i}\right)^{k_{i}}}{\operatorname{det} \gamma_{i}^{k_{i} / 2}}\right) f(z)
$$

as in (2.1); the factor $h(\gamma, z)$ is an automorphy factor of weight $\left(\frac{1}{2}, \ldots, \frac{1}{2}\right)$ defined on the metaplectic cover of $\Gamma_{0}(4 \mathfrak{N})$ by

$$
h(\gamma, z)=\frac{\theta(\gamma z)}{\theta(z)}, \quad \text { where } \theta(z)=\sum_{a \in \mathbb{Z}_{F}} q^{\operatorname{Tr}\left(a^{2} z\right)} .
$$

In this paper, the half-integral weight modular forms we encounter will arise from linear combinations of weighted theta series, as follows.

Let $Q: V \rightarrow F$ be a positive definite quadratic form on an $F$-vector space $V$ with $\operatorname{dim}_{F} V=$ $d$. Let $\Lambda \subset V$ be a (full) $\mathbb{Z}_{F}$-lattice, so $\Lambda$ is a finitely generated $\mathbb{Z}_{F}$-submodule that contains a basis for $V$. Suppose that $\Lambda$ is integral, so $Q(\Lambda) \subseteq \mathbb{Z}_{F}$. Let $\mathfrak{N}$ be the discriminant of $\Lambda$ and suppose that $\mathfrak{N}$ is odd.

A homogeneous polynomial $P(x)=P\left(x_{1}, \ldots, x_{d}\right)$ on $\mathbb{R}^{d}$ is spherical harmonic if

$$
\left(\frac{\partial^{2}}{\partial x_{1}^{2}}+\ldots+\frac{\partial^{2}}{\partial x_{d}^{2}}\right) P=0
$$

For $i=1, \ldots, n$, let $P_{i}(x)$ be a spherical harmonic polynomial on $V \otimes_{v_{i}} \mathbb{R}$ of homogeneous degree $m_{i}$, and let $P(x)=\prod_{i=1}^{n} P_{i}(x)$. We define the theta series associated to $\Lambda$ and $P$ as

$$
\begin{aligned}
\Theta(\Lambda, P): \mathcal{H}^{n} & \rightarrow \mathbb{C} \\
\Theta(\Lambda, P ; z) & =\sum_{x \in \Lambda} P(x) q^{\operatorname{Tr}(Q(x) z)} .
\end{aligned}
$$

Then $\theta$ is a Hilbert modular form of level $4 \mathfrak{N}$, weight $\left(d / 2+m_{i}\right)_{i}$, and quadratic character $\psi$ of conductor dividing $4 \mathfrak{N}[\mathbf{3 6}]$. Note that $\sum_{i} m_{i}$ must be even, or else trivially we have $\Theta(\Lambda, P)=0$. 


\section{Twists of $L$-functions}

For the analytic properties of Hilbert modular forms we use in this section, we refer to Shimura [32]. Let $f \in S_{k}(\mathfrak{N})$ be a Hilbert newform of weight $k$ and level $\mathfrak{N}$, and let $k_{0}=\max \left(k_{1}, \ldots, k_{n}\right)$. Let $T_{\mathfrak{n}} f=a_{\mathfrak{n}} f$ and let $\lambda_{\mathfrak{n}}=a_{\mathfrak{n}} / \sqrt{\mathrm{N}(\mathfrak{n})}^{k_{0}-1}$.

Associated to $f$ is an $L$-function $L(f, s)$ given by the normalized Dirichlet series

$$
L(f, s)=\sum_{\mathfrak{n}} \frac{\lambda_{\mathfrak{n}}}{\mathrm{N}(\mathfrak{n})^{s}}
$$

convergent for $\operatorname{Re} s>1$. Define the completed $L$-function

$$
\Lambda(f, s)=Q^{s / 2}\left(\prod_{i=1}^{n} \Gamma_{\mathbb{C}}\left(s+\left(k_{i}-1\right) / 2\right)\right) \cdot L(f, s)
$$

where $Q=d_{F}^{2} \mathrm{~N}(\mathfrak{N})$ is the conductor and $\Gamma_{\mathbb{C}}(s)=2(2 \pi)^{-s} \Gamma(s)$. Then $\Lambda(f, s)$ has an analytic continuation to $\mathbb{C}$ and satisfies the functional equation

$$
\Lambda(f, s)=w_{f} \Lambda(f, 1-s) \quad \text { with } w_{f}=(-1)^{\left(k_{1}+\ldots+k_{n}\right) / 2} w_{\mathfrak{N}, f},
$$

where $w_{\mathfrak{N}, f}$ is the canonical (Atkin-Lehner) eigenvalue of $f$.

We will be interested in twisting $L$-functions by quadratic characters, which are indexed by fundamental discriminants, as follows. The ring of integers of a quadratic field $K$ is of the form $\mathbb{Z}_{K}=\mathbb{Z}_{F}[x] /\left(x^{2}-t x+n\right)$ with $t, n \in \mathbb{Z}_{F} ;$ the quantity $D=t^{2}-4 n \in \mathbb{Z}_{F} / \mathbb{Z}_{F}^{\times 2}$ is the fundamental discriminant uniquely associated to $K=F(\sqrt{D})$. Consequently, there is a bijection between the set of isomorphism classes of quadratic fields $K / F$ and fundamental discriminants $D$. An element $D \in \mathbb{Z}_{F}$ is a fundamental discriminant if and only if $D$ is a square modulo 4 and minimal with respect to this property under divisibility by squares: that is, we have $D \equiv t^{2}(\bmod 4)$ for some $t \in \mathbb{Z}_{F}$ and, if $D=f^{2} d$ with $d, f \in \mathbb{Z}_{F}$ and $d$ a square modulo 4 , then $f \in \mathbb{Z}_{F}^{\times}$. Let

$$
\mathcal{D}\left(\mathbb{Z}_{F}\right)=\left\{D \in \mathbb{Z}_{F} / \mathbb{Z}_{F}^{\times 2}: D \text { fundamental discriminant and } D \ll 0\right\}
$$

be the set of totally negative fundamental discriminants. For $D \in \mathcal{D}\left(\mathbb{Z}_{F}\right)$, let $\chi_{D}$ be the quadratic character associated to $K=F(\sqrt{D})$.

Suppose that $D$ is coprime to $\mathfrak{N}$. We consider the quadratic twist $f_{D}=f \otimes \chi_{D} \in S_{k}\left(\mathfrak{N} D^{2}\right)$ of $f$ and its associated $L$-function $L\left(f, s, \chi_{D}\right)$ defined by

$$
L\left(f, s, \chi_{D}\right)=L\left(f_{D}, s\right)=\sum_{\mathfrak{n}} \chi_{D}(\mathfrak{n}) \frac{\lambda_{\mathfrak{n}}}{\mathrm{N}(\mathfrak{n})^{s}}
$$

The completed $L$-function $\Lambda\left(f, s, \chi_{D}\right)=\Lambda\left(f_{D}, s\right)$ is defined as in (2.5) with $Q=d_{F}^{2} \mathrm{~N}\left(\mathfrak{N} D^{2}\right)$; it satisfies the functional equation

$$
\Lambda\left(f, s, \chi_{D}\right)=w_{f_{D}} \Lambda\left(f, 1-s, \chi_{D}\right) \quad \text { with } w_{f_{D}}=(-1)^{n} \chi_{D}(\mathfrak{N}) w_{f} .
$$

Definition 2.6. A weight satisfies the parity condition if $\left(k_{1}+\ldots+k_{n}\right) / 2 \equiv n(\bmod 2)$. We say that $D \in \mathcal{D}\left(\mathbb{Z}_{F}\right)$ is permitted if $D$ is coprime to $\mathfrak{N}$ and $\chi_{D}(\mathfrak{q})=w_{\mathfrak{q}, f}$ for all $\mathfrak{q} \| \mathfrak{N}$.

The parity condition ensures that

$$
w_{f}=(-1)^{n} w_{\mathfrak{N}, f},
$$


and $w_{f_{D}}=\chi_{D}(\mathfrak{N}) w_{\mathfrak{N}, f}$. For permitted $D$ we have $\chi_{D}(\mathfrak{N})=w_{\mathfrak{N}, f}$, thus $w_{f_{D}}=1$. Note that the parity condition for parallel weight $k \in 2 \mathbb{Z}_{>0}$ holds when $n$ is even or when $n$ is odd and $k \equiv 2(\bmod 4)$.

To simplify notation, we write $|D|=-D$ when $D$ is totally negative.

ConjeCture 2.8. Let $f \in S_{k}(\mathfrak{N})$ be a Hilbert newform of odd squarefree level $\mathfrak{N}$ such that $k$ satisfies the parity condition. Then there exists a modular form $g(z)=\sum_{\mu \in\left(\mathfrak{d}^{-1}\right)_{+}} c_{\mu} q^{\operatorname{Tr}(\mu z)} \in$ $S_{(k+1) / 2}(4 \mathfrak{N})$ such that for all permitted $D \in \mathcal{D}\left(\mathbb{Z}_{F}\right)$, we have

$$
L\left(f, 1 / 2, \chi_{D}\right)=\kappa_{f} \frac{c_{|D|}(g)^{2}}{\prod_{i=1}^{n}{\sqrt{\left|v_{i}(D)\right|}}^{k_{i}-1}},
$$

where $\kappa_{f} \neq 0$ is independent of $D$. In the case of parallel weight $k$ the denominator in the right-hand side is just $\sqrt{\mathrm{N}(D)}^{k-1}$.

Conjecture 2.8 is known in many cases. It follows in principle from general work of Waldspurger [43] and Shimura [35], but we were not able to extract the explicit statement above: the difficult part is to construct a nonzero form $g$. Baruch-Mao [1] prove Conjecture 2.8 in the case $F=\mathbb{Q}$ (using work of Kohnen for the existence of $g$ ). Xue [46] gives a proof for general $F$ and prime level $\mathfrak{N}=\mathfrak{p}$, provided $L\left(f, \frac{1}{2}\right) \neq 0$; Hiraga-Ikeda [18] treat general $F$ and trivial level $\mathfrak{N}=(1)$. We will discuss in $\S 3$ an algorithm that conjecturally always computes the form $g$ associated to $f \in S_{k}(\mathfrak{N})$ when $L\left(f, \frac{1}{2}\right) \neq 0$ : it is correct as long as $g \neq 0$, by the work of the above authors.

REMARK 2.10. The condition that $\mathfrak{N}$ is odd and squarefree is important here for the purposes of a concise exposition; already in the case $F=\mathbb{Q}$, to relax these hypotheses involves technical complications. Similarly, there are extensions to totally real fundamental discriminants as well, but we do not pursue them here. The restriction to permitted discriminants is necessary; if $(D / \mathfrak{l})=-w_{\mathfrak{l}, f}$ for some prime $\mathfrak{l} \| \mathfrak{N}$ then the coefficient $c_{|D|}$ is trivially zero but $L\left(f, \frac{1}{2}, \chi_{D}\right)$ need not be zero.

\section{Algorithms}

In this section, we discuss algorithms to test the vanishing or nonvanishing of central values $L\left(f, \frac{1}{2}, \chi_{D}\right)$ using the theory of the previous section, encoded as the coefficients of a linear combination of quaternionic theta series. We continue with our notation from the previous section: in particular, let $f \in S_{k}(\mathfrak{N})$ be a Hilbert newform over $F$. Throughout, we employ conventions and algorithms described by Kirschmer-Voight [21, 22]; a general reference for the results on quaternion algebras we will use is Vignéras [42].

As we will be employing an algorithmic version of Conjecture 2.8, we assume that $\mathfrak{N}$ is odd and squarefree and that the weight $k$ satisfies the parity condition (Definition 2.6). Moreover, we make the assumption that $L\left(f, \frac{1}{2}\right) \neq 0$ to work with the simplest construction of the associated half-integral weight form $g$ : for extensions, see Remark 3.2 below.

\section{Brandt module}

The assumption that $L\left(f, \frac{1}{2}\right) \neq 0$ implies, in particular, that $w_{f}=1$. By (2.7), we have $w_{\mathfrak{N}, f}=(-1)^{n} ;$ in other words $\#\left\{\mathfrak{l} \mid \mathfrak{N}: w_{\mathfrak{l}, f}=-1\right\} \equiv n(\bmod 2)$, and it follows that there is a quaternion algebra $B$ over $F$ ramified at all the real places and at primes $\mathfrak{l} \mid \mathfrak{N}$ with $w_{\mathfrak{l}, f}=-1$. Since $\mathfrak{N}$ is squarefree there is an Eichler order $\mathcal{O} \subset B$ with reduced discriminant $\mathfrak{N}$. 
The quaternion algebra $B$ has a unique involution ${ }^{-}: B \rightarrow B$ such that the reduced norm $\operatorname{nrd}(\alpha)=\alpha \bar{\alpha} \in F$ and reduced trace $\operatorname{trd}(\alpha)=\alpha+\bar{\alpha} \in F$ belong to $F$ for all $\alpha \in B$. The discriminant

$$
\Delta(\alpha)=-(\alpha-\bar{\alpha})^{2}=4 \operatorname{nrd}(\alpha)-\operatorname{trd}(\alpha)^{2} \in F
$$

defines a positive definite quadratic form $\Delta: B / F \rightarrow F$.

A right fractional ideal of $\mathcal{O}$ is a finitely generated $\mathbb{Z}_{F}$-submodule $I \subset B$ with $I F=B$ such that $I \mathcal{O} \subseteq I$. The right order of a right fractional ideal $I$ is

$$
\mathcal{O}_{R}(I)=\{x \in B: I x \subseteq I\} .
$$

Left fractional ideals and left orders are defined analogously. A right fractional ideal of $\mathcal{O}$ has $\mathcal{O}_{R}(I)=\mathcal{O}$ if and only if $I$ is (left) invertible, so there exists a left fractional ideal $I^{-1}$ with $\mathcal{O}_{R}\left(I^{-1}\right)=\mathcal{O}_{L}(I)$ such that $I^{-1} I=\mathcal{O}$.

Let $I, J$ be invertible right fractional $\mathcal{O}$-ideals. We say that $I$ and $J$ are in the same right ideal class (or are isomorphic) if there exists an $\alpha \in B^{\times}$such that $I=\alpha J$, or equivalently if $I$ and $J$ are isomorphic as right $\mathcal{O}$-modules. We write $[I]$ for the equivalence class of $I$ under this relation and denote the set of invertible right $\mathcal{O}$-ideal classes by $\mathrm{Cl} \mathcal{O}$. The set $\mathrm{Cl} \mathcal{O}$ is finite and $H=\# \mathrm{ClO}$ is independent of the choice of Eichler order $\mathcal{O}$. Let $I_{1}, \ldots, I_{H}$ be a set of representatives for $\mathrm{Cl} \mathcal{O}$ such that $\operatorname{nrd} I_{i}$ is coprime to $\mathfrak{N}$ for all $i$.

Let $U_{i}$ be the space of spherical harmonic polynomials of homogeneous degree $\left(k_{i}-2\right) / 2$ on $B_{v_{i}} / F_{v_{i}} \cong \mathbb{R}^{3}$ (with respect to $\Delta$ ) and let

$$
W_{k}=\bigoplus_{i=1}^{n} U_{i} .
$$

Then $B^{\times}$(and hence $B^{\times} / F^{\times}$) acts on $W_{k}$ by conjugation on each factor. The space $M_{k}^{B}(\mathfrak{N})$ of quaternionic modular forms of weight $k$ and level $\mathfrak{N}$ for $B$ can be represented as

$$
M_{k}^{B}(\mathfrak{N}) \cong \bigoplus_{i=1}^{H} W_{k}^{\Gamma_{i}}
$$

where $\Gamma_{i}=\mathcal{O}_{L}\left(I_{i}\right)^{\times} / \mathbb{Z}_{F}^{\times}$. By the correspondence of Eichler, Shimizu, and Jacquet-Langlands, $M_{k}^{B}(\mathfrak{N})$ is isomorphic as a Hecke module to a subspace of $\mathrm{M}_{k}(\mathfrak{N})$ of forms that are new at all the primes where $B$ is ramified. In particular, $f$ corresponds to a quaternionic modular form given by $\left(P^{(1)}, \ldots, P^{(H)}\right)$ with $P^{(i)} \in W_{k}^{\Gamma_{i}}$. Algorithms to compute the coefficients $P^{(i)}$ are due to Socrates-Whitehouse [39] and Dembélé [8] and are surveyed in the work of Dembélé-Voight $[10]$.

The form $g \in M_{(k+1) / 2}(4 \mathfrak{N})$ associated to $f$ by Conjecture 2.8 can be (conjecturally) computed as

$$
g(z)=\sum_{i=1}^{H} \frac{1}{\# \Gamma_{i}} \Theta\left(\Lambda^{(i)}, P^{(i)} ; z\right)
$$

where $\Lambda^{(i)}=\mathcal{O}_{L}\left(I_{i}\right) / \mathbb{Z}_{F}$ has quadratic form $\Delta$ and spherical harmonic polynomial $P^{(i)}$. Therefore, to compute the (non)vanishing twists $L\left(f, 1 / 2, \chi_{D}\right)$, encoded in the coefficients $c_{|D|}(g)$, we need only compute the theta series $\Theta\left(\Lambda^{(i)}, P^{(i)}\right)$ with sufficiently many terms and choose a unique representative for each fundamental discriminant.

REMARK 3.2. If we follow this construction with $L(f, 1 / 2)=0$, then the corresponding form has $g=0$ : see Gross [15] and Böcherer-Schulze-Pillot $[\mathbf{3}]$ for the case $F=\mathbb{Q}$ and Xue [46] and Sirolli [38] for general $F$. On the other hand, one expects a nonzero $g$ in Conjecture 2.8; see Mao-Rodriguez-Villegas-Tornaría [24] for an extension of this algorithm to compute $g$ in this case. 


\section{Theta series}

To expand a theta series over $\mathbb{Z}_{F}$ such as in the previous section, we consider the form over $\mathbb{Z}$ given by the trace, as follows. Let $\Lambda$ be an integral $\mathbb{Z}_{F}$-lattice with a positive definite quadratic form $Q$ and a spherical polynomial $P$, and let $\Theta(\Lambda, P)$ be its theta series as in (2.4). We will exhibit a method to compute the expansion of this series over all terms $q^{\nu}$ with $\operatorname{Tr} \nu \leqslant T$.

For each embedding $v_{i}: F \hookrightarrow \mathbb{R}$ the quadratic form $Q: \Lambda \rightarrow \mathbb{Z}_{F}$ yields a positive definite quadratic form $Q_{i}: \Lambda \rightarrow \mathbb{R}$, and the sum

$$
\begin{aligned}
\operatorname{Tr} Q: \Lambda \cong \mathbb{Z}^{d n} & \rightarrow \mathbb{Z} \\
x & \mapsto \operatorname{Tr} Q(x)
\end{aligned}
$$

is a positive definite quadratic form over $\mathbb{Z}$. Then the Fincke-Pohst algorithm [12], based on the LLL lattice reduction algorithm [23], can be used to iterate over the elements of

$$
X_{T}=\{x \in \Lambda: \operatorname{Tr} Q(x) \leqslant T\}
$$

using $O\left(T^{d n / 2}\right)$ bit operations for fixed $\Lambda, Q, P$ as $T \rightarrow \infty$, which is proportional to the volume of the associated region. This allows one to compute the coefficients of

$$
\Theta(\Lambda, P ; z)=c_{0}+\sum_{\nu \in\left(\mathbb{Z}_{F}\right)_{+}} q^{\operatorname{Tr}(\nu z)}
$$

for all $\nu$ with $\operatorname{Tr} \nu \leqslant T$ as

$$
c_{\nu}=\sum_{\substack{x \in X_{T} \\ Q(x)=\nu}} P(x) .
$$

\section{Discriminants}

To test the conjectures highlighted in the introduction, among the coefficients in the theta series computed in the previous subsection we need to find a unique set of representatives for fundamental discriminants with bounded norm (not trace). In this subsection, we explain how to do this.

First, we will need to work with 'balanced' representatives of fundamental discriminants, up to the action of $U_{F}=\mathbb{Z}_{F}^{\times 2}$. To do so, we find a fundamental domain $\Delta$ for the action of $U_{F}$ on $F_{+} \hookrightarrow \mathbb{R}_{>0}^{n}$, and choose the representative $D$ such that $-D$ lies in this fundamental domain. The study of such domains was pioneered by Shintani [37]; see also the exposition by Neukirch [25, § VII.9].

Theorem 3.3. There exists a $\mathbb{Q}$-rational polyhedral cone $\Delta$ that is a finite disjoint union of simplicial cones such that

$$
\mathbb{R}_{>0}^{n}=\bigsqcup_{u \in U_{F}} u \Delta
$$

Shintani gives an effective procedure for computing $\Delta$, but it is quite complicated to carry out in practice. Further algorithms for computing the cone $\Delta$ are given by Okazaki $[\mathbf{2 6}]$ and Halbritter-Pohst [16].

For concreteness, in this subsection we assume that $F$ is a real quadratic field. (Similar arguments work for higher degree fields, including work done for real cubic fields $[\mathbf{1 1}, \mathbf{4 0}]$, but for degree $n=[F: \mathbb{Q}] \geqslant 4$, they are quite a bit more complicated.) In this case, we have $\mathbb{Z}_{F}^{\times 2}=\epsilon^{\mathbb{Z}}$; replacing $\epsilon$ by $1 / \epsilon$, we may assume that $\epsilon_{2}>\epsilon_{1}$, and then $\epsilon$ is unique. Then the Shintani domain $\Delta$ is the cone over the vectors $(1,1)$ and $\left(\epsilon_{1}, \epsilon_{2}\right)$, so $\left(x_{1}, x_{2}\right) \in \Delta$ if and only if $1 \leqslant x_{2} / x_{1}<\epsilon_{2} / \epsilon_{1}$. 
Lemma 3.4. If $x=\left(x_{1}, x_{2}\right) \in \Delta$ has $\mathrm{N}(x)=x_{1} x_{2} \leqslant X$ then

$$
\operatorname{Tr} x=x_{1}+x_{2} \leqslant \operatorname{Tr}(\epsilon) \sqrt{X} .
$$

Proof. By homogeneity, it suffices to prove this for the case $X=1$. Then the maximum value of $\operatorname{Tr} x$ subject to $\mathrm{N}(x)=1$ and $x \in \Delta$ occurs at the vertex $\left(\epsilon_{1}, \epsilon_{2}\right)$.

With Lemma 3.4 in hand, to compute the coefficients $c_{|D|}(g)$, one for each $D \in \mathcal{D}\left(\mathbb{Z}_{F} ; X\right)$, we apply the method of the previous subsection with $T=\gamma_{F} \sqrt{X}$ where $\gamma_{F}=\operatorname{Tr}(\epsilon)$ and select only those with $-D \in \Delta$. We can further restrict to fundamental discriminants by factoring $D \mathbb{Z}_{F}$ to ensure that its odd part is squarefree, the exponent of an even prime is $\leqslant 3$, and finally that $D / e^{2}$ is not a square modulo 4 for all even nonassociate prime elements $e$. In a similar way, we can enumerate all permitted $D \in \mathcal{D}\left(\mathbb{Z}_{F} ; X\right)$ using the unary theta series $Q(x)=x^{2}$ on $\mathbb{Z}_{F}$.

REMARK 3.5. For a totally real field $F$ of degree $n=[F: \mathbb{Q}]$, by similar reasoning and the arithmetic-geometric mean, there exists $\gamma_{F}>n$ depending only on $F$ such that for all $x \in \Delta$, we have

$$
n \mathrm{~N}(x)^{1 / n} \leqslant \operatorname{Tr}(x) \leqslant \gamma_{F} \mathrm{~N}(x)^{1 / n},
$$

that is, $\operatorname{Tr}(x)=\Theta\left(\mathrm{N}(x)^{1 / n}\right)$. (In fact, $\gamma_{F}=\max _{\epsilon}|\operatorname{Tr}(\epsilon)|$ for $\epsilon$ a ray of the Shintani cone.) So for the purposes of testing our conjectures, one can use either trace or norm, and for higher degree fields, the former is much simpler to implement.

REMARK 3.6. The algorithms above are used for experimental purposes, so we do not give a precise running time for them; however, we can give a rough idea of the running time (which bears out in practice): for a fixed field $F$, the time to compute $\left\{c_{|D|}(g): D \in \mathcal{D}\left(\mathbb{Z}_{F} ; X\right)\right\}$ for a Hilbert modular newform $f \in S_{k}(\mathfrak{N})$ over $F$ is governed by computing theta series, which is roughly

$$
O\left((\mathrm{~N} \mathfrak{N})\left(X^{1 / n}\right)^{3 n / 2}\right)=O\left((\mathrm{~N} \mathfrak{N}) \cdot X^{3 / 2}\right)
$$

because $\operatorname{Tr}(D)=O\left(X^{1 / n}\right)$ for $-D \in \Delta$ and $\operatorname{dim} S_{k}(\mathfrak{N})=O(\# \mathrm{Cl} \mathcal{O})=O(\mathrm{~N} \mathfrak{N})$ by Eichler's mass formula [42, Corollaire V.2.5].

\section{Example}

The Hilbert modular form of parallel weight 2 and smallest level norm over $F=\mathbb{Q}(\sqrt{5})$ is the form $f$ whose label is $2 \cdot 2 \cdot 5 \cdot 1-31.1$-a (see $\S 5$ for more on the labeling system), and we take this form as an example to illustrate the above algorithms. Let $w=(1+\sqrt{5}) / 2$, so $w$ satisfies $w^{2}-w-1=0$ and $\mathbb{Z}_{F}=\mathbb{Z}[w]$. Let $\mathfrak{N}=(5 w-2)$, so $N \mathfrak{N}=31$. Then there is a unique form in $S_{2}(\mathfrak{N})$; it has rational Hecke eigenvalues

$$
a_{(2)}=-3, a_{(2 w-1)}=-2, a_{(3)}=2, a_{(3 w-2)}=4, \ldots
$$

and is neither CM nor base change. By the Eichler-Shimura construction, this form corresponds to the isogeny class of the elliptic curve

$$
E: y^{2}+x y+w y=x^{3}+(w+1) x^{2}+w x
$$

via $\# E\left(\mathbb{Z}_{F} / \mathfrak{p}\right)=\mathrm{N} \mathfrak{p}+1-a_{\mathfrak{p}}$ for $\mathfrak{p} \neq \mathfrak{N}$.

The $L$-function $L(f, s)$ has conductor $Q=5^{2} \cdot 31=775$ and $L\left(f, \frac{1}{2}\right)=0.3599289 \ldots \neq 0$; the Atkin-Lehner eigenvalue is $w_{\mathfrak{N}, f}=w_{f}=1$. Like any form of parallel even weight $k$ over a real quadratic field, the form $f$ satisfies the parity condition $2 k \equiv 0(\bmod 4)$. 
We find a quaternion algebra ramified only at the two real places of $F$ to be $B=((-1,-1) / F)$, with Eichler order of level $\mathfrak{N}$ given by

$$
\mathcal{O}=\mathbb{Z}_{F} \oplus \mathbb{Z}_{F}(5 w-2) i \oplus \mathbb{Z}_{F} \frac{(w+1)+(w+8) i+j}{2} \oplus \mathbb{Z}_{F} \frac{w+(w+25) i+k}{2} .
$$

There are two right ideal classes in $\mathcal{O}$, with the class $\left[I_{1}\right]=[\mathcal{O}]$ and the nontrivial class represented by the ideal

$$
I_{2}=(w+3) \mathcal{O}+\frac{(w+11)+(w+70)+j}{2} \mathcal{O}
$$

of reduced norm $\operatorname{nrd}(I)=(3 w-2)$ itself of norm 11 . We have $\# \Gamma_{1}=\#\left(\mathcal{O}_{1}^{\times} / \mathbb{Z}_{F}^{\times}\right)=5$ and $\# \Gamma_{2}=3$. In parallel weight 2 , the weight space $W_{k}=\mathbb{C}$ is trivial, and we find that accordingly $M_{2}^{B}(\mathfrak{N})$ has dimension 2 as a $\mathbb{C}$-vector space. The eigenform $f$ corresponds to the eigenvector $5\left[I_{1}\right]-3\left[I_{2}\right]$, so we have $P^{(1)}=5$ and $P^{(2)}=-3$.

We have $\mathcal{O}_{L}(\mathcal{O})=\mathcal{O}$, and the discriminant form $\Delta$ on $\Lambda^{(1)}=\mathcal{O} / \mathbb{Z}_{F}$ gives a theta series

$$
\begin{aligned}
\Theta\left(\Lambda^{(1)} ; z\right) & =\sum_{v \in \Lambda^{(1)}} q^{\operatorname{Tr}(\Delta(v) z)} \\
& =1+q^{\operatorname{Tr}((-w+3) z)}+q^{(\operatorname{Tr}(w+2) z)}+0 q^{\operatorname{Tr}(3 z)}+q^{\operatorname{Tr}((4 w+3) z)}+\ldots
\end{aligned}
$$

The terms $\nu=0,-4 w+12,4 w+8$ correspond to nonfundamental discriminants. In a similar way we compute $\Theta\left(\Lambda^{(1)} ; z\right)$ and then compute

$$
\begin{aligned}
g(z) & =\Theta\left(\Lambda^{(1)}, P^{(1)} ; z\right)+\Theta\left(\Lambda^{(2)}, P^{(2)} ; z\right)=\frac{5}{5} \Theta\left(\Lambda^{(1)} ; z\right)-\frac{3}{3} \Theta\left(\Lambda^{(2)} ; z\right) \\
& =q^{\operatorname{Tr}((w+2) z)}+q^{\operatorname{Tr}((-w+3) z)}-q^{\operatorname{Tr}(3 z)}-q^{\operatorname{Tr}((3 w+3) z)}+q^{\operatorname{Tr}((-3 w+6) z)}+q^{\operatorname{Tr}((4 w+3) z)}+\ldots
\end{aligned}
$$

We confirm that the quadratic twists of $E$ by the discriminants $D=-w-2, w-3,-3,-3 w-$ $3,3 w-6,-4 w-3$ all have rank 0 , consistent with this series; we find out first vanishings at $D=8 w-43,-9 w-38$, and the corresponding twists indeed have rank 2 .

\section{Conjectures about central values of $L$-functions}

Random matrix theory has proved useful in refining conjectures related to the low-lying zeros of $L$-functions [19, 20]. In work of Conrey-Keating-Rubinstein-Snaith [4], the following basic question was considered. Let $f \in S_{k}(N)$ be a newform with rational integer coefficients. For how many fundamental discriminants $D$ with $|D| \leqslant X$ does the twisted $L$-function $L\left(f, s, \chi_{D}\right)$ vanish at the center of the critical strip? In a collection of papers $[\mathbf{2 4}, \mathbf{2 9}, \mathbf{3 0}]$, a number of variants of this problem were considered: the weight of $f$ was allowed to vary, the level of $f$ was allowed to be composite, and so on.

\section{Simple heuristics}

To motivate the power of $X$ in our Conjectures 1.3 and 1.5, we start with two simple heuristics that follow a similar coarse reasoning and ignore logarithmic factors. Let

$$
\mathcal{N}_{f}(X)=\#\left\{D \in \mathcal{D}(\mathbb{Z} ; X): \chi_{D}(N)=-w_{f} \text { and } L\left(f, 1 / 2, \chi_{D}\right)=0\right\}
$$

count the number of vanishing twists of $f$ with sign +1 . If $k=2$, then $f$ corresponds to an elliptic curve $E$ over $\mathbb{Q}$ of conductor $N$, and so assuming the (weak) Birch-Swinnerton-Dyer conjecture, the function $N_{f}(X)$ counts the proportion of twists of $E$ with rank at least two. The function $\mathcal{N}_{f}(X)$ has a heuristic estimate attributed to Sarnak, as follows. As discussed in 
$\S 2$, Conjecture 2.8 relates the central value of the $D$ th twist of $f$ to the coefficient $c_{|D|}(g)$ of a particular half-integer weight modular form $g$ attached to $f$ according to the formula

$$
L\left(f, 1 / 2, \chi_{D}\right)=\kappa_{f} \frac{c_{|D|}(g)^{2}}{\sqrt{|D|}} .
$$

The Ramanujan-Petersson bound on the coefficients of $g$ is $|D|^{1 / 4+\varepsilon}$ for all $\varepsilon>0$, and so, if $c_{|D|}(g)$ takes a value in the range $\left[0,|D|^{1 / 4}\right]$ for $|D| \leqslant X$ that is not too far from uniform, then it should take the value 0 approximately $X^{3 / 4+\varepsilon}$ of the time.

The same heuristic extends to higher weight and to the case of Hilbert modular forms, as follows. Let $f$ be a Hilbert modular form of parallel weight $k$ over a field $F$ of degree $n=[F: \mathbb{Q}]$. The Lindelöf hypothesis (see for example, Baruch-Mao [1, Conjecture 1.4]) implies that the central values of any family of quadratic twist $L$-functions satisfying the Riemann hypothesis have

$$
L\left(f, 1 / 2, \chi_{D}\right)=O\left(|\mathrm{~N}(D)|^{\varepsilon}\right)
$$

for all $\varepsilon>0$ as $|\mathrm{N}(D)| \rightarrow \infty$. By Conjecture 2.8, the bound (4.2) is equivalent to

$$
\left|c_{|D|}(g)\right|=O\left(|\mathrm{~N}(D)|^{(k-1) / 4+\varepsilon}\right)
$$

for all $\varepsilon>0$, so this would also be asserted by the Ramanujan-Petersson bound for half-integral weight Hilbert modular forms.

Now, consider the context of Conjecture 1.3. If for each $D \in \mathcal{D}\left(\mathbb{Z}_{F} ; X\right)$ the coefficient $c_{|D|}(g)$ has a distribution in $\left[0,|\mathrm{~N}(D)|^{(k-1) / 4}\right]$ that is not too far from uniform, then the expected number of times when the value is 0 is approximately $X^{1-(k-1) / 4+\varepsilon}$, since $\mathcal{D}\left(\mathbb{Z}_{F} ; X\right)=\Theta(X)$. We note that this bound is independent of $n=[F: \mathbb{Q}]$.

On the other hand, consider the case considered in Conjecture 1.5 where we twist only by twist $D \in \mathcal{D}(\mathbb{Z} ; X)$. If $c_{|D|}(g)$ is still distributed in a way not too far from uniform, then since

$$
|\mathrm{N}(D)|^{(k-1) / 4+\varepsilon}=|D|^{n(k-1) / 4+n \varepsilon}
$$

then it should vanish about $X^{1-n(k-1) / 4+\varepsilon}$ for all $\varepsilon>0$.

The power of $\log$

We now pay attention to the power of $\log X$ appearing in our conjectures, and for this refinement we must dig a bit deeper. Consider first the case $F=\mathbb{Q}$ and weight $k$. We follow the heuristic $[4,19]$ prescribed by random matrix theory, verified by the large scale computation of central values of $L$-functions twisted by a quadratic character over $\mathbb{Q}$; see also further work by Watkins [44]. In this theory, low-lying zeros of $L$-functions are related to values of characteristic polynomials of random matrices of $\mathrm{SO}(2 m)$ and we deduce

$$
\operatorname{Prob}\left[L\left(f, 1 / 2, \chi_{D}\right)=0\right] \sim C_{f} 2^{\lambda(D, f)} \frac{(\log |D|)^{3 / 8}}{|D|^{(k-1) / 4}}
$$

for a constant $C_{f}$. Here $2^{\lambda(D, f)}$ is a multiplicative function that increases with the number of prime divisors of $D$ and depends on the arithmetic and geometric nature of $f$. We assume, too, that for some constant $b_{f}$, we have $2^{\lambda(D, f)} \approx(\log D)^{b_{f}}$ on average. For example, DelaunayWatkins [7] for the case of an elliptic curve $E$ (so $F=\mathbb{Q}$ and $k=2$ ) predict the exponent $b_{E}$ where $b_{E}^{\prime}=1, \frac{\sqrt{2}}{2}, \frac{1}{3}, \frac{\sqrt{2}}{2}-\frac{1}{3}$ depending on properties of the mod 2 Galois representations occurring for elliptic curves in the isogeny class of $E$ and, roughly speaking, define $2^{\lambda(D, f)}$ to be the product of the Tamagawa numbers $g_{p}$ for $p \mid D$. A further refinement could be achieved 
by considering $2^{\lambda(D, f)}$ to be related to the power of 2 that divides the coefficient $c_{|D|}(g)$ of the half-integer weight form that corresponds to $f$ as in Conjecture 2.8 over $\mathbb{Q}$ when this conjecture is a theorem. To find the total number of vanishings, we sum the probability in (4.3) over all discriminants up to $X$.

In the setting of Hilbert modular forms we arrive at a formula in an identical way. As far as we can tell, the random matrix theory arguments do not see the field over which the Hilbert modular form is defined; they only care about the $L$-function and its functional equation. It is reasonable then to suppose that we have

$$
\operatorname{Prob}\left[L\left(f, 1 / 2, \chi_{D}\right)=0\right] \sim C_{f} 2^{\lambda_{F}(D, f)} \frac{(\log |\mathrm{N}(D)|)^{A_{n}}}{|D|^{(k-1) / 4}}
$$

in analogy with (4.3), where $A_{n}$ is a constant that depends only on the degree of the field (arising from the normalization of $m$ in $\mathrm{SO}(2 m) ; A_{1}=\frac{3}{8}$ ) and $2^{\lambda_{F}(D, f)}$ is a multiplicative function that increases with the number of primes in $\mathbb{Z}_{F}$ dividing the ideal $(D)$. For Conjecture 1.3, we suppose that $2^{\lambda_{F}(D, f)}$ for $D \in \mathcal{D}\left(\mathbb{Z}_{F}\right)$ is, for some $b_{f}$, on average roughly of size $(\log \mathrm{N}(D))^{b_{f}}$ and so we have

$$
\begin{aligned}
\mathcal{N}_{f}\left(\mathbb{Z}_{F} ; X\right) & \sim \sum_{D \in \mathcal{D}\left(\mathbb{Z}_{F} ; X\right)} 2^{\lambda_{F}(D, f)} \frac{(\log |\mathrm{N}(D)|)^{A_{n}}}{|\mathrm{~N}(D)|^{(k-1) / 4}} \\
& \sim \sum_{D \in \mathcal{D}\left(\mathbb{Z}_{F} ; X\right)}(\log |\mathrm{N}(D)|)^{b_{f}} \frac{(\log |\mathrm{N}(D)|)^{A_{n}}}{|\mathrm{~N}(D)|^{(k-1) / 4}} \sim C_{f} X^{(1-(k-1) / 4)}(\log X)^{b_{f}+A_{n}} .
\end{aligned}
$$

For example, if $\lambda_{F}(D, f)=\omega_{F}(D)$, we have $b_{f}=1$ : the congruent number curve for $F=\mathbb{Q}$ is an example where $\lambda_{\mathbb{Q}}(D, f)=\omega(D)$.

Now consider the sum

$$
\sum_{D \in \mathcal{D}(Z ; X)} 2^{\lambda_{F}(D, f)} \frac{(\log |\mathrm{N}(D)|)^{A_{n}}}{|\mathrm{~N}(D)|^{(k-1) / 4}}
$$

as required by Conjecture 1.5. Now we must consider $\lambda_{F}(D)$ with $D \in \mathbb{Z}$. Let $e_{F}$ be the expected number of distinct primes $\mathfrak{p}$ in $\mathbb{Z}_{F}$ dividing $(p)$ for $p \in \mathbb{Z}$ prime. For example, for $F$ quadratic we have $e_{F}=2\left(\frac{1}{2}\right)+1\left(\frac{1}{2}\right)=\frac{3}{2}$, and for $F$ of degree $n$ with $\operatorname{Gal}(F / \mathbb{Q}) \cong S_{n}$, we have $e_{F}=1+\frac{1}{2}+\ldots+1 / n$ is the $n$th harmonic number. Then $2^{\lambda_{F}(D, f)} \sim 2^{e_{F} \lambda_{\mathbb{Q}}(D, f)} \approx \log (D)^{b_{f} e_{F}}$ where the $b_{f}$ is as in the beginning of this section. Substituting in this estimate, and using that $\mathrm{N}(D)=D^{n}$ for $D \in \mathbb{Z}$, we are led to conjecture that

$$
\mathcal{N}_{f}(\mathbb{Z} ; X) \sim C_{f, \mathbb{Z}} X^{(1-n(k-1) / 4)}(\log X)^{b_{f} e_{F}+A_{n}},
$$

as predicted by Conjecture 1.5, with $b_{f, \mathbb{Z}}=b_{f} e_{F}$.

\section{Computations}

In this section we describe the results of the computations resulting from our implementation of the algorithms in $\S 3$. When describing a Hilbert modular form we use the labelling found in the LMFDB [9]. For example, the label 2.2.5.1-[2,4]-37.1-b means the form over the field $F$ with label 2.2.5.1 ('degree 2 with 2 real places of discriminant 5 numbered 1', that is, $F=\mathbb{Q}(\sqrt{5}))$ with weight $k=(2,4)$, level norm 31 and ideal numbered 1 , and isogeny class $b$. If $k=(2, \ldots, 2)$ is parallel weight 2 , we will suppress it and write simply 2.2.5.1-31.1-a, for example. 


\section{Verification}

To make sure that we got the statement of Conjecture 2.8 exactly correct in the cases we care about, we computationally verified the statement as written by comparing the central values we get by computing them directly using Icalc [31] and by using the coefficients of the theta series computed with the algorithms described below. Consider the ratio $D / 3 \cdot L\left(f, \frac{1}{2}, \chi_{D}\right) / L\left(f, \frac{1}{2}, \chi_{-3}\right)$ where $D \in \mathbb{Z}$. By Conjecture 2.8 , this ratio should be the square of an integer, namely the square of the ratio of the corresponding coefficients of the associated half-integral modular form. For the forms 2.2.5.1-31.1-a and 2.2.13.1-4.1-a, we checked the twists $D=-3,-7,-11,-19,-23,-43$.

We also verified for the form 2.2.5.1-31.1-a and all $|\mathrm{N}(D)|<10000$ that the vanishing of $c_{|D|}$ matched the vanishing of $L\left(E, 1, \chi_{D}\right)$ with $E$ as in (3.7) using Magma. (Similar verification was performed by Sirolli [38].)

\section{Overview of examples}

A Hilbert modular form $f$ over $F$ is a base change (BC) if there exists a proper subfield $F^{\prime} \subsetneq F$ and a Hilbert modular form $f^{\prime}$ over $F^{\prime}$ of some weight and level such that the base change of $f^{\prime}$ (when it exists) to $F$ is equal to $f$ (up to quadratic twist).

For each of the examples below, we tabulated data related to vanishings of central values of twists and data related to the distributions of values of coefficients:

(1) $F$ quadratic, $k=2(2.2 .5 .1-31.1-\mathrm{a}$, neither CM nor BC; 2.2.8.1-9.1-a, BC but not $\mathrm{CM} ; 2.2 .24 .1-1.1-\mathrm{a}, \mathrm{BC}$ and $\mathrm{CM})$;

(2) $F=\mathbb{Q}(\sqrt{5}), k=4(2 \cdot 2 \cdot 5 \cdot 1-[4,4]-11.1-a$, neither BC nor CM); and

(3) $F$ cubic, $k=2(3.3 .49 .1-41.1-a$, neither BC nor CM).

(We did not collect enough data for a $\mathrm{BC}$ and $\mathrm{CM}$ form; a potential candidate is the form 2.2.24.1-1.1-a.)

\section{A first example}

We dedicate particular attention to the data related to the form 2.2.5.1-31.1-a which we gave as an example at the end of $\S 3$. In Table 1, we give some timings to provide some idea of how long our computations of this example took and also to provide data related to Conjectures 1.3 and 1.5. We study the vanishings $\mathcal{N}_{f}\left(\mathbb{Z}_{F} ; X\right)$ and $\mathcal{N}_{f}(\mathbb{Z} ; X)$ as defined in (1.2) and (1.4). See also Figure 1 for a graphical representation of these data as well as some data related to the distributions of the Fourier coefficients of the Shimura lift of $f$.

Because the power $b_{f}$ of $\log X$ is still uncertain, we appealed to another prediction made by random matrix theory (RMT): for a prime $\mathfrak{q} \nmid \mathfrak{N}$, we define

TABLE 1. Statistics for the Hilbert modular form 2.2.5.1-31.1-a over $\mathbb{Q}(\sqrt{5})$.

\begin{tabular}{llrrrc}
\hline$X$ & Time & $\# \mathcal{D}\left(\mathbb{Z}_{F} ; X\right)$ & $\mathcal{N}_{f}\left(\mathbb{Z}_{F} ; X\right)$ & $\# \mathcal{D}(\mathbb{Z} ; X)$ & $\mathcal{N}_{f}(\mathbb{Z} ; X)$ \\
\hline $10^{2}$ & $0.07 \mathrm{~s}$ & 3 & 0 & & \\
$10^{3}$ & $0.4 \mathrm{~s}$ & 41 & 0 & & \\
$10^{4}$ & $5 \mathrm{~s}$ & 439 & 41 & & \\
$10^{5}$ & $1 \mathrm{~m} 40 \mathrm{~s}$ & 4481 & 397 & & \\
$10^{6}$ & $47 \mathrm{~m}$ & 44865 & 3173 & & \\
$10^{7}$ & $46 \mathrm{~h}$ & 448667 & 24748 & 387 & 50 \\
$10 \cdot 10^{7}$ & & 4486620 & 183100 & 1229 & 120 \\
$15 \cdot 10^{7}$ & $1.5 \mathrm{y}$ & 6729969 & 259525 & 1509 & 141 \\
\hline
\end{tabular}




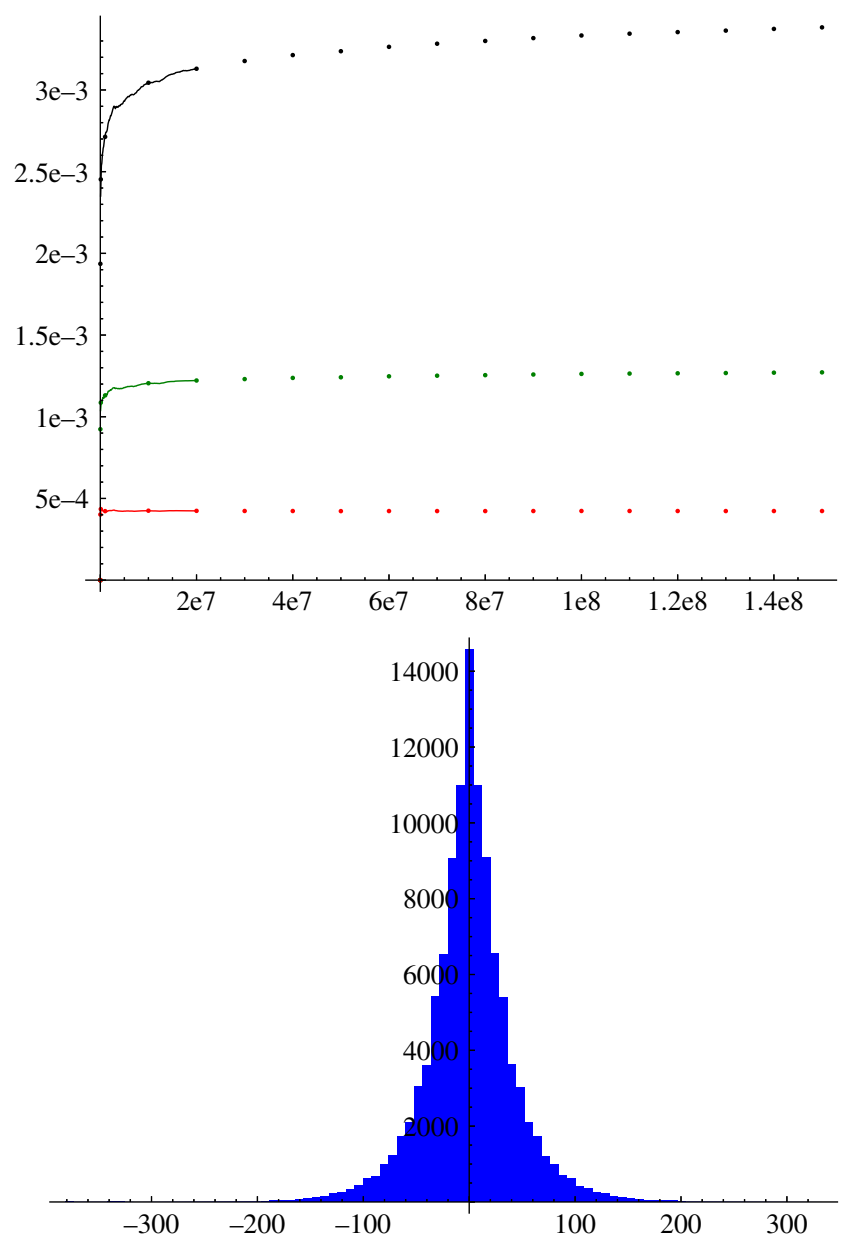

Figure 1. The top figure is $\mathcal{N}_{f}\left(\mathbb{Z}_{F}, X\right) /\left(X^{3 / 4}(\log X)^{e_{f}}\right)$ for the form 2.2.5.1-31.1-a and for various powers of log. The powers of $\log$ are $e_{f}=\frac{11}{8}$ (shown in black), $e_{f}=\frac{41}{24}$ (shown in green) and $e_{f}=\frac{25}{12}$ (shown in red). The powers of $\log$ were determined as follows: $e_{f}=\frac{11}{8}$ is the prediction analogous to the one made by Delaunay-Watkins for curves with full 2-torsion over $\mathbb{Q}$ but over $F ; e_{f}=\frac{25}{12}$ is the least-squares fit of the data and $e_{f}=\frac{41}{24}$ comes from fitting the data to the computation of vanishings in a slice (see Remark 3.5) and comparing it with the vanishings up to $X$. The bottom figure is the distribution of the values of the coefficients $c_{|D|}(g)$ that corresponds to $f$ as in Conjecture 2.8, for $\mathrm{N}(D)<2 \cdot 10^{7}$.

$$
\begin{gathered}
\mathcal{N}_{f}\left(\mathbb{Z}_{F}, \mathfrak{q}, \pm 1 ; X\right)=\#\left\{D \in \mathcal{D}\left(\mathbb{Z}_{F} ; X\right): \chi_{D}(\mathfrak{N})=(-1)^{n} w_{f} \text { and } L\left(f, 1 / 2, \chi_{D}\right)=0\right. \\
\text { and } \left.\left(\frac{D}{\mathfrak{q}}\right)= \pm 1\right\}
\end{gathered}
$$

as well as

$$
R_{\mathfrak{q}}(f ; X)=\frac{\mathcal{N}_{f}\left(\mathbb{Z}_{F}, \mathfrak{q},+1 ; X\right)}{\mathcal{N}_{f}\left(\mathbb{Z}_{F}, \mathfrak{q},-1 ; X\right)} \quad \text { and } \quad R_{\mathfrak{q}}(f)=\lim _{X \rightarrow \infty} R_{\mathfrak{q}}(f ; X)
$$

Then one expects that the limit $R_{\mathfrak{q}}(f)$ exists $[4$, Conjecture 2] and

$$
R_{\mathfrak{q}}(f)=\sqrt{\frac{N \mathfrak{q}+1-a_{\mathfrak{q}}}{N \mathfrak{q}+1+a_{\mathfrak{q}}}} .
$$


In Table 2, we computed the ratios (5.1) for several primes; the agreement is good, which gives a general indication that the predictions of RMT are relevant in this context.

REMARK 5.2. We did one extra experiment with this example by taking a slice of coefficients farther out for the example. In order to do this efficiently, we took a 'trace slice', not a 'norm slice'; according to Remark 3.5, we expect qualitatively the same behavior, but potentially a different constant. We computed vanishing statistics for all negative fundamental discriminants $D$ with

$$
3 \sqrt{10^{9}}<|\operatorname{Tr}(D)| \leqslant 3 \sqrt{10^{9}+10^{6}}
$$

(here $\gamma_{F}=3$ for $F=\mathbb{Q}(\sqrt{5})$ ); according to Remark 3.5, this corresponds to the range with

$$
10^{9}<|\mathrm{N}(D)| \leqslant 10^{9}+10^{6}
$$

up to a constant factor. We find that there are 1761 vanishings out of 75688 for twists over $\mathbb{Z}_{F}$ and 0 vanishings out of 3 for twists over $\mathbb{Z}$.

We also computed the ratios (5.1) inside this slice: we also found weak agreement, for example,

$$
\frac{\mathcal{N}_{f}\left(\mathbb{Z}_{F} ; \mathfrak{q} ;+1 ; 10^{9}+10^{6}\right)-\mathcal{N}_{f}\left(\mathbb{Z}_{F} ; \mathfrak{q} ;+1 ; 10^{9}\right)}{\mathcal{N}_{f}\left(\mathbb{Z}_{F} ; \mathfrak{q} ;-1 ; 10^{9}+10^{6}\right)-\mathcal{N}_{f}\left(\mathbb{Z}_{F} ; \mathfrak{q} ;-1 ; 10^{9}\right)}=\frac{822}{536}=1.533 \approx 1.414
$$

for $\mathrm{N}(\mathfrak{q})=5$, and similarly for $\mathrm{N}(\mathfrak{q})=9,11,11$ we found $\frac{625}{877}=0.713 \approx 0.816, \frac{609}{874}=0.697 \approx$ 0.707 , and $\frac{903}{597}=1.513 \approx 1.414$.

\section{Vanishings}

We mention further experimental results related to vanishings. In Figures $1-4$ we provide a graphical representation of the ratio $\mathcal{N}_{f}\left(\mathbb{Z}_{F}, X\right) /\left(X^{3 / 4}(\log X)^{11 / 8}\right)$ for a variety of Hilbert modular forms of weight 2 . These plots all qualitatively get flat at about the same rate, suggesting to us that they are obeying the same qualitative law, the law from Conjecture 1.3. In Figure 5 we provide a graphical representation of $\mathcal{N}_{f}\left(\mathbb{Z}_{F}, X\right) /\left(X^{1 / 4}(\log X)^{11 / 8}\right)$ for a form of weight 4 , as well as some data related to the distribution of the Fourier coefficients of the Shimura lift of $f$.

Our experiments provide convincing evidence for Conjecture 1.3, but cannot help us find the power of log despite having a massive amount of data; by comparison, we can provide almost no evidence for Conjecture 1.5 since we collected only a tiny amount of data. On the other hand, the simple heuristics we provided in $\S 4$, assuming that Conjecture 1.3 is correct, give strong evidence for Conjecture 1.5.

TABLE 2. Congruence ratios for 2.2.5.1-31.1-a over $\mathbb{Q}(\sqrt{5})$.

\begin{tabular}{rcc}
\hline$N \mathfrak{q}$ & $R_{\mathfrak{q}}\left(f ; 2 \cdot 10^{7}\right)$ & $\sqrt{\frac{N \mathfrak{q}+1-a_{\mathfrak{q}}}{N \mathfrak{q}+1+a_{\mathfrak{q}}}}$ \\
\hline 5 & $20925 / 14986=1.396$ & 1.414 \\
9 & $18237 / 22481=0.811$ & 0.817 \\
11 & $17293 / 24674=0.701$ & 0.707 \\
11 & $24899 / 17140=1.453$ & 1.414 \\
19 & $19955 / 24802=0.805$ & 0.817 \\
19 & $24847 / 20015=1.241$ & 1.225 \\
29 & $23955 / 22424=1.068$ & 1.069 \\
29 & $24040 / 22294=1.078$ & 1.069 \\
\hline
\end{tabular}



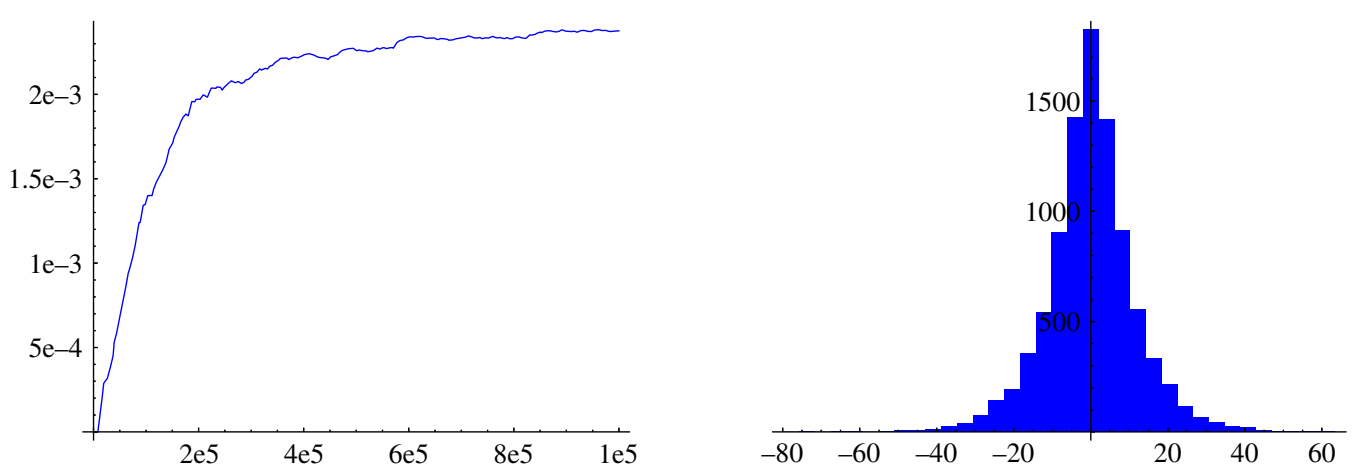

Figure 2. The figure on the left shows $\# N_{f}\left(\mathbb{Z}_{F}, X\right) /\left(X^{3 / 4}(\log X)^{11 / 8}\right)$ for the form 2.2.8.1-9.1-a and the figure on the right shows the distribution of the values of the coefficients $c_{|D|}(g)$ that correspond to $f$ as in Conjecture 2.8 .
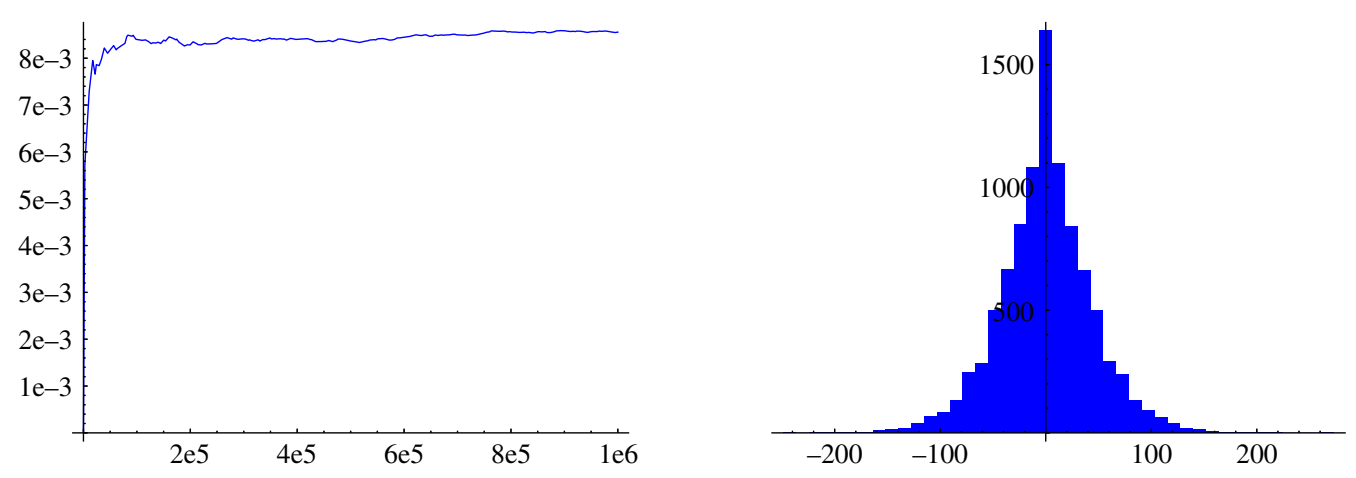

Figure 3. The figure on the left shows $\# N_{f}\left(\mathbb{Z}_{F}, X\right) /\left(X^{3 / 4}(\log X)^{11 / 8}\right)$ for the form 2.2.24.1-1.1-a and the figure on the right shows the distribution of the values of the coefficients $c_{|D|}(g)$ that correspond to $f$ as in Conjecture 2.8.
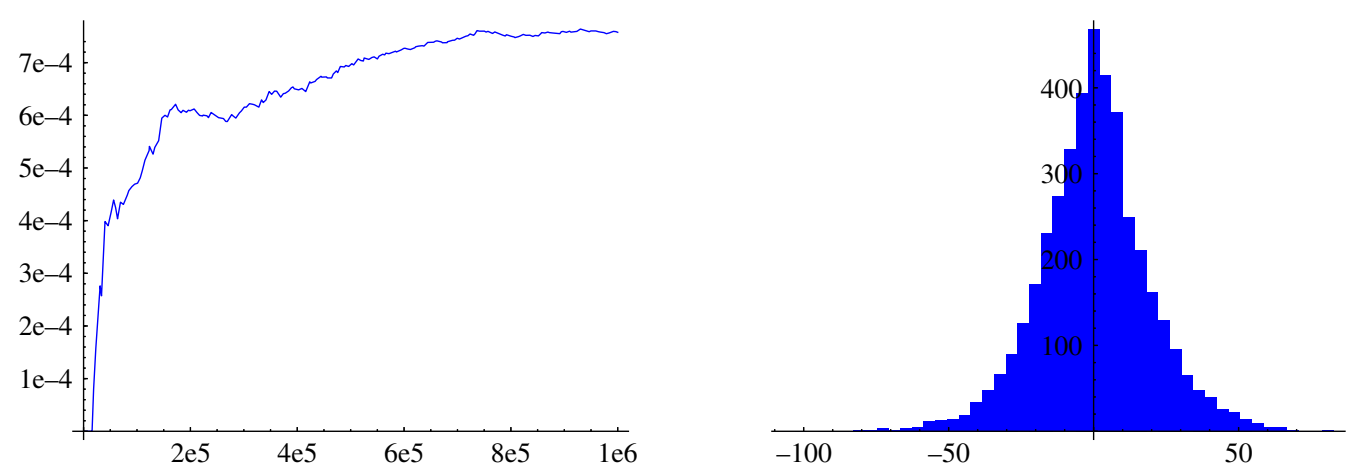

Figure 4. The figure on the left shows $\# N_{f}\left(\mathbb{Z}_{F}, X\right) /\left(X^{3 / 4}(\log X)^{11 / 8}\right)$ for the form 3.3.49.1-41.1-a and the figure on the right shows the distribution of the values of the coefficients $c_{|D|}(g)$ that correspond to $f$ as in Conjecture 2.8. 

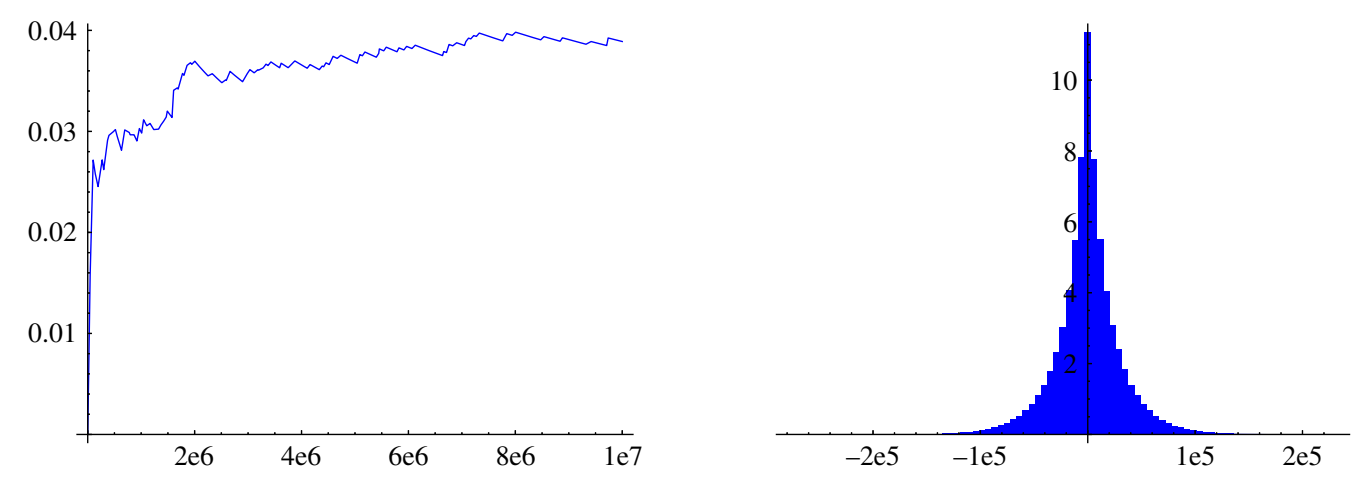

Figure 5. The figure on the left shows $\# N_{f}\left(\mathbb{Z}_{F}, X\right) /\left(X^{1 / 4}(\log X)^{11 / 8}\right)$ for the form 2.2.5.1-[4,4]-11.1-a and the figure on the right shows and the distribution of the values of the coefficients $c_{|D|}(g)$ that correspond to $f$ as in Conjecture 2.8.

\section{Distribution of coefficients}

We computed and stored the coefficients of the Shimura lifts of the examples mentioned above for two reasons. First, while the data related to vanishings discussed in the previous section grows on the order of $X^{3 / 4}$, the number of coefficients grows on the order of $X$. So, we automatically have more data at our disposal to compare with the predictions of random matrix theory $[4,5]$. Second, the distributions of Fourier coefficients of modular forms of even integer weight are related to the Sato-Tate conjecture; given a modular form $f$ the distribution of its Fourier coefficients depends on, for example, whether or not the modular form is CM. The distributions of the Fourier coefficients of the half-integral weight forms mentioned above are also plotted in Figures 1-5 and they all exhibit qualitatively the same behavior, independent of whether or not the form whose Shimura lift we are considering is CM or not.

The data we compute are consistent with the random matrix theory predictions as described in [5] and the distributions we compute match the predictions well.

\section{Remaining questions}

This paper just scratches the surface of this topic, and many questions remain.

(1) What can be said about twists over a totally real field with other signatures? In particular, are the asymptotics the same for discriminants $D$ such that $F(\sqrt{D})$ is of mixed signature? (Such extensions are a genuinely new phenomenon over $F \neq \mathbb{Q}$.)

(2) Do the same asymptotics apply when the narrow class number of $F$ is bigger than 1, or does the class group pose an obstruction?

(3) What happens when the modular form $f$ has coefficients in a field $K$ larger than $\mathbb{Q}$ ? For weight $k=2$, such a form corresponds to an isogeny class of abelian varieties of $\mathrm{GL}_{2}$-type defined over $F$. More care must be taken in the discretization step in this situation, since it must be performed with respect to the ring of integers of $K$ embedded as a lattice.

Acknowledgements. The authors would like to thank Brian Conrey and Ariel Pacetti for helpful discussions, as well as Mark Watkins and the anonymous referees for several comments and corrections. 


\section{References}

1. E. M. BARUCh and Z. MAO, 'Central value of automorphic L-functions', Geom. Funct. Anal. 17 (2007) no. 2, 333-384.

2. W. Bosma, J. Cannon and C. Playoust, 'The Magma algebra system. I. The user language', J. Symbolic Comput. 24 (1997) no. 3-4, 235-265; Computational algebra and number theory (London, 1993).

3. S. Böcherer and R. Schulze-Pillot, 'On a theorem of Waldspurger and on Eisenstein series of Klingen type', Math. Ann. 288 (1990) no. 3, 361-388.

4. J. B. Conrey, J. P. Keating, M. O. Rubinstein and N. C. Snaith., 'On the frequency of vanishing of quadratic twists of modular L-functions', Number theory for the millennium, I (Urbana, IL, 2000) (A K Peters, Natick, MA, 2002) 301-315.

5. J. B. Conrey, J. P. Keating, M. O. Rubinstein and N. C. Snaith, 'Random matrix theory and the Fourier coefficients of half-integral-weight forms', Experiment Math. 15 (2006) no. 1, 67-82.

6. C. David, J. Fearnley and H. Kisilevsky, 'Vanishing of $L$-functions of elliptic curves over number fields', Ranks of elliptic curves and random matrix theory, London Mathematical Society Lecture Note Series 341 (Cambridge University Press, Cambridge, 2007) 247-259.

7. C. Delaunay and M. Watkins, 'The powers of logarithm for quadratic twists', Ranks of elliptic curves and random matrix theory, London Mathematical Society Lecture Note Series 341 (Cambridge University Press, Cambridge, 2007) 189-193.

8. L. DembéLé, 'Quaternionic Manin symbols, Brandt matrices, and Hilbert modular forms', Math. Comp. 76 (2007) no. 258, 1039-1057.

9. L. Démbéle, S. Donnelly and J. Voight, LMFDB: L-function and modular form database, 2013, http://www.lmfdb.org.

10. L. DembéLÉ and J. Voight, 'Explicit methods for Hilbert modular forms', Elliptic curves, Hilbert modular forms and Galois deformations (Birkhäuser, Basel, 2013) 135-198.

11. F. Diaz y Diaz and E. Friedman, 'Colmez cones for fundamental units of totally real cubic fields', J. Number Theory 132 (2012) no. 8, 1653-1663.

12. U. Fincke and M. Pohst., 'Improved methods for calculating vectors of short length in a lattice, including a complexity analysis', Math. Comp. 44 (1985) no. 170, 463-471.

13. E. Freitag, Hilbert modular forms (Springer, Berlin, 1990).

14. E. Z. Goren, Lectures on Hilbert modular varieties and modular forms, CRM Monograph Series 14 (American Mathematical Society, Providence, RI, 2002). With the assistance of Marc-Hubert Nicole.

15. B. H. Gross, 'Heights and the special values of $L$-series', Number theory (Montreal, Que., 1985), CMS Conference Proceedings 7 (American Mathematical Society, Providence, RI, 1987) 115-187.

16. U. Halbritter and M. E. Pohst, 'On lattice bases with special properties', J. Théor. Nombres Bordeaux 12 (2000) no. 2, 437-453; Colloque International de Théorie des Nombres (Talence, 1999).

17. W. B. Hart, G. Tornaría and M. Watkins, 'Congruent number theta coefficients to 1012', Algorithmic number theory (Springer, 2010) 186-200.

18. K. Hiraga and T. IKEDA, 'On the Kohnen plus space for Hilbert modular forms of half-integral weight I', Compos. Math. 149 (2013) no. 12, 1963-2010.

19. J. P. Keating and N. C. Snaith, 'Random matrix theory and $L$-functions at $s=1 / 2$ ', Comm. Math. Phys. 214 (2000) no. 1, 91-110.

20. J. P. Keating and N. C. Snaith, 'Random matrix theory and $\zeta(1 / 2+i t)$ ', Comm. Math. Phys. 214 (2000) no. $1,57-89$.

21. M. Kirschmer and J. Voight, 'Algorithmic enumeration of ideal classes for quaternion orders', SIAM J. Comput. 39 (2010) no. 5, 1714-1747.

22. M. Kirschmer and J. Voight, 'Corrigendum: Algorithmic enumeration of ideal classes for quaternion orders', SIAM J. Comput. 41 (2012) no. 3, 714; MR 2592031.

23. A. K. Lenstra, H. W. Lenstra JR. and L. Lovász, 'Factoring polynomials with rational coefficients', Math. Ann. 261 (1982) no. 4, 515-534.

24. Z. Mao, F. Rodriguez-Villegas and G. Tornaría, 'Computation of central value of quadratic twists of modular L-functions', Ranks of elliptic curves and random matrix theory, London Mathematical Society Lecture Note Series 341 (Cambridge University Press, Cambridge, 2007) 273-288.

25. J. Neukirch, Algebraic number theory, Grundlehren der Mathematischen Wissenschaften (Fundamental Principles of Mathematical Sciences) 322 (Springer, Berlin, 1999). Translated from the 1992 German original and with a note by Norbert Schappacher, with a foreword by G. Harder.

26. R. OKAZAKI, 'On an effective determination of a Shintani's decomposition of the cone $\mathbf{R}_{+}^{n}$, , J. Math. Kyoto Univ. 33 (1993) no. 4, 1057-1070.

27. A. Pacetti and G. Tornaría, 'Examples of the Shimura correspondence for level $p^{2}$ and real quadratic twists', Ranks of elliptic curves and random matrix theory, London Mathematical Society Lecture Note Series 341 (Cambridge University Press, Cambridge, 2007) 289-314.

28. A. Pacetti and G. Tornaría, 'Shimura correspondence for level $p^{2}$ and the central values of $L$-series', J. Number Theory 124 (2007) no. 2, 396-414. 
29. A. Pacetti and G. Tornaría, 'Computing central values of twisted $L$-series: the case of composite levels', Experiment. Math. 17 (2008) no. 4, 459-471.

30. H. Rosson and G. Tornaría, 'Central values of quadratic twists for a modular form of weight 4', Ranks of elliptic curves and random matrix theory, London Mathematical Society Lecture Note Series 341 (Cambridge University Press, Cambridge, 2007) 315-321.

31. M. O. Rubinstein, lcalc: The L-function calculator, a C++ class library and command line program, 2008, http://www.math.uwaterloo.ca/ mrubinst.

32. G. Shimura, 'The special values of the zeta functions associated with Hilbert modular forms', Duke Math. J. 45 (1978) no. 3, 637-679.

33. G. Shimura, 'On Eisenstein series of half-integral weight', Duke Math. J. 52 (1985) no. 2, 281-314.

34. G. Shimura, 'On Hilbert modular forms of half-integral weight', Duke Math. J. 55 (1987) no. 4, 765-838.

35. G. Shimura, 'On the Fourier coefficients of Hilbert modular forms of half-integral weight', Duke Math. J. 71 (1993) no. 2, 501-557.

36. G. Shimura, 'On the transformation formulas of theta series', Amer. J. Math. 115 (1993) no. 5, 1011-1052.

37. T. Shintani, 'On evaluation of zeta functions of totally real algebraic number fields at non-positive integers', J. Fac. Sci. Univ. Tokyo Sect. IA Math. 23 (1976) no. 2, 393-417.

38. N. Sirolli, Preimages for the Shimura map on Hilbert modular forms, Preprint, 2012, arXiv:1208.4011 [math.NT].

39. J. Socrates and D. Whitehouse, 'Unramified Hilbert modular forms, with examples relating to elliptic curves', Pacific J. Math. 219 (2005) no. 2, 333-364.

40. E. Thomas and A. T. VAsquez, 'On the resolution of cusp singularities and the Shintani decomposition in totally real cubic number fields', Math. Ann. 247 (1980) no. 1, 1-20.

41. G. VAN DER GeER, Hilbert modular surfaces, Ergebnisse der Mathematik und ihrer Grenzgebiete (3) [Results in Mathematics and Related Areas (3)] 16 (Springer, Berlin, 1988).

42. M.-F. Vignéras, 'Arithmétique des algèbres de quaternions', Lecture Notes in Mathematics 800 (Springer, Berlin, 1980).

43. J.-L. WAldspurger, 'Sur les coefficients de Fourier des formes modulaires de poids demi-entier', J. Math. Pures Appl. (9) 60 (1981) no. 4, 375-484.

44. M. Watkins, 'On elliptic curves and random matrix theory', J. Théor. Nombres Bordeaux 20 (2008) no. 3, 829-845.

45. M. WatKins, 'Some heuristics about elliptic curves', Experiment Math. 17 (2008) no. 1, 105-125.

46. H. Xue, 'Central values of $L$-functions and half-integral weight forms', Proc. Amer. Math. Soc. 139 (2011) no. $1,21-30$.

\author{
Nathan C. Ryan \\ Department of Mathematics \\ Bucknell University \\ Lewisburg, PA 17837 \\ USA \\ nathan.c.ryan@gmail.com \\ John Voight \\ Department of Mathematics \\ Dartmouth College \\ 6188 Kemeny Hall \\ Hanover, NH 03755 \\ USA \\ jvoight@gmail.com
}

\author{
Gonzalo Tornaría \\ Centro de Matemática \\ Universidad de la República \\ 11400 Montevideo \\ Uruguay \\ tornaria@cmat.edu.uy
}

